\title{
REVIEW
}

\section{Tissue engineering in urethral reconstruction—an update}

\author{
Altaf Mangera $^{1}$ and Christopher R Chapple ${ }^{2}$ \\ The field of tissue engineering is rapidly progressing. Much work has gone into developing a tissue engineered urethral graft. Current \\ grafts, when long, can create initial donor site morbidity. In this article, we evaluate the progress made in finding a tissue engineered \\ substitute for the human urethra. Researchers have investigated cell-free and cell-seeded grafts. We discuss different approaches to \\ developing these grafts and review their reported successes in human studies. With further work, tissue engineered grafts may facilitate \\ the management of lengthy urethral strictures requiring oral mucosa substitution urethroplasty.
}

Asian Journal of Andrology (2013) 15, 89-92; doi:10.1038/aja.2012.91; published online 8 October 2012

Keywords: reconstructive urology; tissue engineering; urethra; urethroplasty

\section{INTRODUCTION TO URETHRAL RECONSTRUCTION}

The urethra functions as a channel permitting the passage of urine out of the bladder. Strictures occur most commonly due to a scarring process involving the vascular tissue of the corpus spongiosum leading to ischaemic spongiofibrosis in the urethra. ${ }^{1}$ Occasionally, a specific cause such as lichen sclerosis (BXO) may be identified, although the majority are considered idiopathic iatrogenic strictures, which are on the increase principally due to the increased number of urethral procedures being undertaken.

Management of strictures usually starts with less invasive techniques such as urethral dilatation and urethrotomy, and progresses to more invasive procedures such as anastomotic and substitution urethroplasty. ${ }^{2}$ Urethrotomy and dilatation are standard procedures which are readily available and minimally invasive. The stricture is incised or split respectively and it is hoped that healing will occur without restenosis. Steenkamp and colleagues ${ }^{3}$ have reported that the success of urethrotomy at one year if the stricture was less than $2 \mathrm{~cm}$ was $60 \%$, but only $20 \%$ if the stricture was longer than $4 \mathrm{~cm}$. Heyns et al. ${ }^{4}$ have shown that a second urethrotomy has shown benefit only in up to $40 \%$ of individuals up to 48-month follow-up; however if the stricture recurred early ( $<3$ months), then the failure rate was $100 \%$ by 48 months.

Anastomotic urethroplasty has been shown to have excellent success approaching $90 \%$ or more for anterior urethral strictures, particularly involving the bulbar urethra. ${ }^{5}$ There are various techniques, but briefly; anastomotic urethroplasty involves removal of the stricture and simple anastomosis of the urethral ends. This is only feasible in the event of shorter strictures, measuring up to $6 \mathrm{~cm}$. Longer strictures require a flap or graft procedure to fill the defect. Dubey et al. ${ }^{6}$ have shown that there was no difference in the success rate of flaps or grafts, but the former led to greater morbidity. Therefore grafts are the preferred choice for substitution. Oral mucosa has become the graft of choice due to its ease of access, resilience and low donor site morbidity for small grafts. ${ }^{7}$ The grafts also take well and have a privileged immunology. Non-hairy skin can also be used as a graft, but is contra-indicated in patients with lichen sclerosis. Hairy skin, particularly scrotal skin, has been used for many years and clinical experience has suggested that this leads on to a number of problems. Hair in the neourethra is a source of infection and also can lead on to stone formation. While clinical experience has suggested that this is the case, it is surprising that in the literature, there is almost no comment on the complications of this from clinical studies using scrotal skin, emphasizing the reporting bias which one sees in the surgical literature. It is as a consequence of these complications that hairy skin, in particular scrotal skin, has been dropped from the therapeutic armamentarium now that other options are available.

\section{Requirement for tissue engineered grafts}

The use of oral mucosa is limited by its availability. A combination of buccal (cheek), lingual (tongue) and labial (lip) mucosa may be utilized. Patients with reduced mouth opening or previous oral surgery may have less oral tissue for use. In addition, longer grafts carry a greater morbidity. While intraoperative haemorrhage, post-operative infection, pain, swelling, damage to salivary ducts, limited oral opening, altered sensation or numbness, scarring and even deformity have been reported, this remains a technique remarkably free of serious long term sequelae. The risk of donor site morbidity is increased in smokers, tobacco chewers and those with poor oral hygiene. ${ }^{8}$ In these cases, there may be a need for a substitute for oral mucosa.

The ideal substitute should take well, not undergo contraction, fibrosis or rejection, be impermeable, cheap and have good handling characteristics. There have been a lot of clinical case studies reported to date using various techniques for substitution urethroplasty. In this article, we will review the progress made in the field of tissue engineering with regard to urethral substitution.

\section{TISSUE ENGINEERED GRAFTS}

Tissue engineering is a rapidly progressing discipline. Many tissue engineered grafts have been used for urethral reconstruction. 
Essentially urethral grafts can be separated into those that contain living autologous cells and those that are cell-free. The latter include grafts obtained from cadaveric or animal sources. This tissue is then treated to make it completely cell free. The resultant biological matrix is then implanted. A good vascular bed is needed to allow take and infiltration of host cells. As a rule, these techniques would only be expected to be particularly successful for substituting short urethral defects.

In contrast, cellularized grafts contain a matrix populated with autologous cells. These cells are obtained from a small biopsy which is harvested from the patient. The cells are cultured, expanded and seeded on to the matrix. The matrix containing cells is then implanted onto the host bed. Certain techniques may be utilized in vitro to create stratification of the graft, such as raising the grafts to the air-liquid interface. This causes stratification of keratinocytes to form an impermeable layer at the top of the graft. Cell seeded grafts also require a vascular wound bed for cell survival.

\section{Cell-free tissue engineered grafts}

Clinically acellular grafts have not shown universally good success rates, with small reported series often with short follow-up. elKassaby et al. ${ }^{9}$ have shown take of cadaveric acellular bladder matrix in eight patients out of nine in whom a good vascular bed was present, but in patients with poor wound beds, only two out of six were successful. The mean follow-up was 25 months. The authors reported no cases of fibrosis.

Palminteri et al. ${ }^{10}$ have used porcine small intestinal submucosa (SIS) as an acellular urethroplasty matrix with $94 \%$ success in the bulbar urethra at short term. Cystoscopy revealed acellular areas of graft and success rates were poorer for penile procedures, due to fibrosis occurring in the grafts. Fiala et al. ${ }^{11}$ have reported $80 \%$ success rates at medium-term follow-up (31 months) with SIS grafts. Failures occurred within 6 months and were more common in penile stricture repair. Mantovani et al. ${ }^{12}$ have reported good urethral patency rates at six months follow-up in four patients with a porcine SIS graft. Similarly, Donkov et al. ${ }^{13}$ have shown good success rates with SIS in short strictures between $3 \mathrm{~cm}$ and $4 \mathrm{~cm}$ in eight of nine patients at short term follow-up. Lin et al. ${ }^{14}$ have reported $75 \%$ success rates at 45 months in 16 patients receiving acellular dermal matrix.

The longest follow up data available (71 months) is from Palminteri et al., ${ }^{15}$ where 25 men underwent patch graft urethroplasty using SIS. Failure, defined as need for re-intervention, was described in $24 \%$ of patients. Interestingly, all cases where a long graft $(>4 \mathrm{~cm})$ was required failed.

In contrast, Hauser et al. ${ }^{16}$ reported poorer results with acellular SIS graft. Four out of five patients required re-intervention due to stricture recurrence at a mean of 12 months. In another study, endoscopic placement of SIS grafts failed in six of eight patients due to re-stricturing which occurred within three months of surgery. ${ }^{17}$ In the latter study, however, tubularized grafts were used which are known to lead to poorer results in urethroplasty procedures. ${ }^{5}$ This is principally thought to be due to a lack of native urethral interposition and thus subsequent scarring can occur circumferentially without interruption.

The perceived advantage of acellular biological matrices is the potential of providing a ready-made, 'off the shelf' scaffold that allows host cell ingrowth. ${ }^{18}$ SIS in particular has been shown to release growth factors such as vascular endothelial growth factor to increase angiogenesis. ${ }^{19}$ The grafts become replaced by host tissue and are degraded according to the amount of cross linking in the graft pre-implantation. ${ }^{20}$ It was feared that graft degradation may lead to a risk of pouching and diverticulum formation; however, this has not been shown to be the case. $^{21}$ However, there have been concerns regarding the length of unseeded graft which may take because Dorin et al. ${ }^{22}$ have shown that unseeded grafts greater $0.5 \mathrm{~cm}$ in length did not allow new transitional epithelium formation in rabbit urethras.

From the above data, it is suggested that acellular grafts may not show promise in patients with long strictures $(>4 \mathrm{~cm})$, who have had failed previous urethroplasty or have poor blood supply in their stricture bed. The failures that are reported tended to occur early and are most likely a consequence of failure of graft population by epithelial cells leading to graft fibrosis and re-stricturing. Clearly, while some positive results have been reported-very little information is available on long-term follow-up and these grafts are unlikely to be appropriate for long strictures.

\section{Cellular tissue engineered grafts}

Autologous cell seeded grafts have been proposed to overcome the deficiencies seen with acellular grafts, since success is not dependent on ingrowth of epithelial cells. The early and limited clinical results obtained with cell seeded grafts show some promise. The drawbacks of a cell-seeded graft are that it requires a period of cell culture in a clean room laboratory and at present is not suitable as an 'off the shelf product. There are also cost and time implications. The cost of a cellularized graft is six-fold of a non-cellularized graft. No costeffectiveness analysis has been reported for these grafts and this may be a critical determinant of whether these grafts enter clinical practice. The time limitations posed by these grafts also make planning of biopsy and surgery imperative. There is a time lag of 2-4 weeks between biopsy and implantation. Once the cellularized graft has been prepared, the surgeon has up to 4 days to implant the graft with any delay leading to increased chance of graft failure. However, the cellular composition and mechanical properties of cell seeded grafts can be approximated better to match urethral tissue and duplicate oral mucosa.

Researchers have reported different cell-seeded grafts. Feng et al. ${ }^{23}$ have described a study of four different scaffold materials assessing mechanical properties and cell attachment to the various seeded grafts. From bladder submucosa, SIS, corpus spongiosum matrix and polyglycolic acid, the corpus spongiosum matrix was reported to have the best mechanical properties, supported cells well and allowed the most cellular infiltration in a rabbit model.

Tissue engineered oral mucosa was firstly reported in five men in $2008 .{ }^{24}$ Cadaveric dermis was seeded with oral fibroblasts and keratinocytes obtained from a small oral biopsy 2 weeks prior. All patients in this study had complex strictures secondary to lichen sclerosis and therefore, were representative of the population in whom tissue engineered grafts are likely to be most beneficial. Initial graft take was $100 \%$; however, at 3 years, three patients had a patent urethra after some form of instrumentation. Intervention was required due to graft contracture. The outcomes must be placed into the context of the challenging patient population being investigated, with graft contracture rates being much higher in cases of lichen sclerosis. Methods to reduce graft contraction in vitro have shown that glutaraldehyde and graft restraint can reduce contraction. ${ }^{25}$ This may help lead to grafts with less propensity to contract.

Other studies creating tissue engineered oral mucosa have described cellularisation of acellular bladder matrix grafts seeded with autologous oral cells. ${ }^{26}$ This work has only reached the in vitro stage using rabbit oral mucosa cells, but the important finding is the biocompatibility of bladder acellular matrix with oral mucosa cells. More 
recently, an abstract reported 10 cases of small strictures between 1 and $3 \mathrm{~cm}$ receiving tissue engineered buccal mucosa. Patients donated a small oral biopsy followed by urethroplasty 3 weeks later. ${ }^{27}$ Urethrography at 3 weeks revealed a wide watertight urethra in five patients and no patients had any donor site complications. This technology now needs to be evaluated in patients who have longer strictures, who would benefit from it most.

Other efforts have included creating a tissue engineered urothelium as opposed to tissue engineered oral mucosa. Autologous urothelial cells obtained from bladder washings have been cultured and expanded in the laboratory and thereafter, transplanted back for repair of hypospadias deformity in a paediatric population. ${ }^{28}$ Results were encouraging, but the authors acknowledge that more work needs to be carried out to improve the tissue engineering techniques. Issues which need addressing include increasing the yield and quality of cells from bladder washings. It is suggested that cells obtained from bladder washings are mostly terminally differentiated and have low multiplying and differentiating potential. Coculture systems also need to be developed which aid isolated autologous cells to multiply and differentiate.

Similarly, Nagele et al. ${ }^{29}$ have described a tissue engineered multilayered urothelium expanded from bladder washings. The authors report that cultures were established from $55 \%$ of specimens, of which $62.5 \%$ formed monolayers. This translates to only one in three patients who had bladder washings taken via a catheter had tissue that could be re-implanted. Despite this low yield, this method of cell extraction is less traumatic than a bladder biopsy.

A third methodology used by Fu et al. ${ }^{30}$ has successfully attached foreskin epidermal cells on an acellular rabbit bladder matrix. In a rabbit urethral model at 6 months, urethrography revealed restricturing when acellular bladder submucosa matrix was utilized, but this was not the case when the same matrix was cultured with foreskin epidermal cells. Grafts which were seeded showed greater stratification of epithelium and increased vascularisation. Using corpus spongiosum matrices, Feng et al. ${ }^{31}$ have shown a coculture system formed the best tissue engineered urothelium. The authors used lingual keratinocytes and corporal smooth muscle cells. These showed the least fibrosis and greatest organisation of muscle fibre bundles as well as no re-stricturing. With these techniques, human studies are evidently warranted.

Continuing with coculture, Raya-Rivera et al. ${ }^{32}$ have taken bladder biopsies from five boys from which epithelial and muscle cells were expanded and seeded on a polyglycolic acid: poly-lactide-co-glycolic acid scaffold. In this study, cystourethroscopy was performed up to 72 months and biopsies up to 36 months; these revealed that the implants had similar histological make-up to native urethral tissue and the reconstructed urethras also had good patency. Although this methodology has shown promise, it does require an invasive bladder biopsy, which was taken suprapubically, carrying its own risks. Whether this is acceptable to patients requires a larger study.

\section{CONCLUSIONS}

The search for a tissue-engineered urethral graft has gained some momentum in recent years. There are many different approaches ranging from unseeded scaffolds, single cell-seeded scaffolds and stratified cultured epithelium. The role of each graft may vary between individual clinical situations. For instance, a short primary bulbar stricture with a good wound bed may be treated with an acellular graft, but the question has to be asked as to whether this patient would be better managed by an anastomotic urethroplasty which has a high success rate. Conversely, longer strictures are probably best treated by cellularized grafts; and in this context, the availability of the technology versus the ease of harvesting oral mucosa for strictures of up to $5 \mathrm{~cm}$ in length has to be questioned. The potential role of tissueengineered urethral substitution probably lies in the context of lengthy strictures where there is a paucity of native tissue, e.g., lengthy lichen sclerosis strictures, lack of oral mucosa such as following failed prior substitution, where the available tissue has been utilized. Current evidence is based on small observational studies. Therefore, larger prospective studies are required in patients with strictures longer than $5 \mathrm{~cm}$ who lack an oral mucosa alternative. In the first instance, with long strictures, acellular and cellular grafts need to be compared in terms of convenience, availability, success rates and cost effectiveness. If these show promise, then a study comparing the use of tissue engineered grafts versus oral mucosa will be indicated.

\section{COMPETING FINANCIAL INTERESTS}

The authors declare no competing financial interests.

1 Warwick RT. Urethral surgery. In: Mundy AR, editor. Current Operative Surgery: Urology. London: Balliere Tindall; 1988. pp160-218.

2 Mangera A, Chapple C. Management of anterior urethral stricture: an evidence-based approach. Curr Opin Urol 2010; 20: 453-8.

3 Steenkamp JW, Heyns CF, de Kock ML. Internal urethrotomy versus dilation as treatment for male urethral strictures: a prospective, randomized comparison. J Urol 1997; 157: 98-101.

4 Heyns CF, Steenkamp JW, de Kock ML, Whitaker P. Treatment of male urethral strictures: is repeated dilation or internal urethrotomy useful? J Urol 1998; 160: 356-8.

5 Mangera A, Patterson JM, Chapple CR. A systematic review of graft augmentation urethroplasty techniques for the treatment of anterior urethral strictures. Eur Urol 2011; 59: 797-814.

6 Dubey D, Vijjan V, Kapoor R, Srivastava A, Mandhani A et al. Dorsal onlay buccal mucosa versus penile skin flap urethroplasty for anterior urethral strictures: results from a randomized prospective trial. J Urol 2007; 178: 2466-9.

7 Barbagli G, Fabri F, Romano G, Michele D, Lazerri M. Evaluation of early, late complications and patient satisfaction in 300 patients who underwent oral graft harvesting from a single cheek using a standardised technique in a referral center experience. J Urol supp/ 2009; 181: 14

8 Sinha RJ, Singh V, Sankhwar SN, Dalela D. Donor site morbidity in oral mucosa graft urethroplasty: implications of tobacco consumption. BMC Urol 2009; 9: 15.

9 el-Kassaby A, Aboushwareb T, Atala A. Randomized comparative study between buccal mucosal and acellular bladder matrix grafts in complex anterior urethral strictures. J Urol 2008; 179: 1432-6.

10 Palminteri E, Berdondini E, Colombo F, Austoni E. Small intestinal submucosa (SIS) graft urethroplasty: short-term results. Eur Urol 2007; 51: 1695-701.

11 Fiala R, Vidlar A, Vrtal R, Belej K, Student V. Porcine small intestinal submucosa graft for repair of anterior urethral strictures. Eur Urol 2007; 51: 1702-8.

12 Mantovani F, Trinchieri A, Mangiarotti B, Nicola M, Castelnuovo $C$ et al. [Reconstructive urethroplasty using porcine acellular matrix: preliminary results.] Arch Ital Urol Androl 2002; 74: 127-8. Italian.

13 Donkov II, Bashir A, Elenkov CH, Panchev PK. Dorsal onlay augmentation urethroplasty with small intestinal submucosa: modified Barbagli technique for strictures of the bulbar urethra. Int J Urol 2006; 13: 1415-7.

14 Lin J, Hao JR, Jin J, Deng SM, Hu J et al. Homologous dermal acellular matrix graft for urethral reconstruction in man (report of 16 cases). Zhonghua Yi Xue Za Zhi 2005; 85: 1057-9. Chinese.

15 Palminteri E, Berdondini E, Fusco F, de NC, Salonia A. Long-term results of small intestinal submucosa graft in bulbar urethral reconstruction. Urology 2012; 79: 695-701.

16 Hauser S, Bastian PJ, Fechner G, Muller SC. Small intestine submucosa in urethral stricture repair in a consecutive series. Urology 2006; 68: 263-6.

17 le Roux PJ. Endoscopic urethroplasty with unseeded small intestinal submucosa collagen matrix grafts: a pilot study. J Urol 2005; 173: 140-3.

18 Badylak S, Liang A, Record R, Tullius R, Hodde J. Endothelial cell adherence to small intestinal submucosa: an acellular bioscaffold. Biomaterials 1999; 20: 2257-63.

19 Hodde JP, Record RD, Liang HA, Badylak SF. Vascular endothelial growth factor in porcine-derived extracellular matrix. Endothelium 2001; 8: 11-24.

20 Santucci RA, Barber TD. Resorbable extracellular matrix grafts in urologic reconstruction. Int Braz J Urol 2005; 31: 192-203.

21 Mantovani F, Tondelli E, Cozzi G, Abed El Rahman D, Spinelli MG et al. [Reconstructive urethroplasty using porcine acellular matrix (SIS): evolution of the grafting technique and results of 10-year experience.] Urologia 2011; 78: 92-7. Italian.

22 Dorin RP, Pohl HG, de Filippo RE, Yoo JJ, Atala A. Tubularized urethral replacement with unseeded matrices: what is the maximum distance for normal tissue regeneration? World J Urol 2008; 26: 323-6. 
23 Feng C, Xu YM, Fu Q, Zhu WD, Cui L et al. Evaluation of the biocompatibility and mechanical properties of naturally derived and synthetic scaffolds for urethral reconstruction. J Biomed Mater Res A 2010; 94: 317-25.

24 Bhargava S, Patterson JM, Inman RD, MacNeil S, Chapple CR. Tissue-engineered buccal mucosa urethroplasty-clinical outcomes. Eur Urol 2008; 53: 1263-9.

25 Patterson JM, Bullock AJ, MacNeil S, Chapple CR. Methods to reduce the contraction of tissue-engineered buccal mucosa for use in substitution urethroplasty. Eur Urol 2011; 60: 856-61.

26 Li C, Xu Y, Song L, Cui L, Yin S. [A preliminary experimental study on urethral reconstruction using tissue engineered oral mucosa.] Zhongguo Xiu Fu Chong Jian Wai Ke Za Zhi 2008; 22: 1242-5. Chinese.

27 Ram-Liebig G, Engel O, Schwaiger B, Reiss P, Pfalzgraf D et al. Tissue-engineered buccal mucosa urethroplasty. Outcome of our first 10 patients. Eur Urol Supp/2012; e621-621a.
28 Fossum M, Nordenskjold A. Tissue-engineered transplants for the treatment of severe hypospadias. Horm Res Paediatr 2010; 73: 148-52.

29 Nagele U, Maurer S, Feil G, Bock C, Krug J et al. In vitro investigations of tissueengineered multilayered urothelium established from bladder washings. Eur Urol 2008; 54: 1414-22.

30 Fu Q, Deng CL, Liu W, Cao YL. Urethral replacement using epidermal cell-seeded tubular acellular bladder collagen matrix. BJU Int 2007; 99: 1162-5.

31 Feng C, Xu YM, Fu Q, Zhu WD, Cui L. Reconstruction of three-dimensional neourethra using lingual keratinocytes and corporal smooth muscle cells seeded acellular corporal spongiosum. Tissue Eng Part A 2011; 17: 3011-9.

32 Raya-Rivera A, Esquiliano DR, Yoo JJ, Lopez-Bayghen E, Soker S et al. Tissueengineered autologous urethras for patients who need reconstruction: an observational study. Lancet $2011 ; 377$ : 1175-82. 